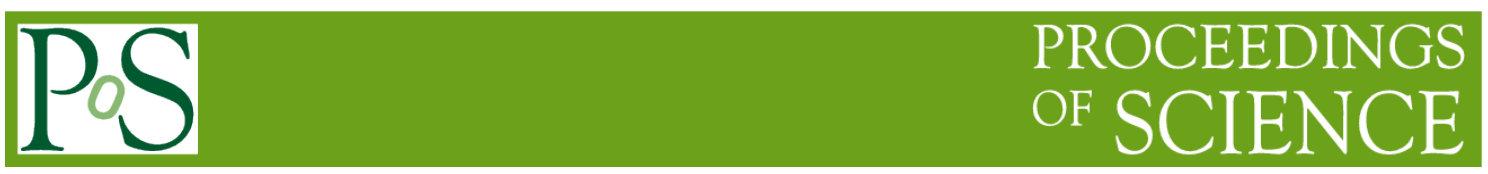

\title{
DBBC3: AntArr Project
}

\section{G. Tuccari, S. Buttaccio}

INAF-Istituto di Radioastronomia

Ctr.da Renna, Noto SR, Italy and

Max Planck Institute fuer Radioastronomie

Auf dem Hugel 69, Bonn, Germany

E-mail: g.tuccarieira.inaf.it

\section{W. Alef ${ }^{1}$, M. Wunderlich}

Max-Planck-Institut für Radioastronomie

Auf dem Hügel 69, Bonn, Germany

E-mail: a lefempifr-bonn.mpg.de

\section{S. Buttaccio}

INAF-Istituto di Radioastronomia

Ctr.da Renna, Noto SR, Italy

\section{Tornatore}

DICA Politectico

Milano, Italy

E-mail: vincenza.tornatore@polimi.it

Low frequency bands in a traditional VLBI networks suffer not only from RFI but also from the low efficiency of the radio telescopes. Dedicated arrays have to be used which have to maintain all the constraints of a VLBI station, e.g., phase stability. With this aim a project with an alternative application of the DBBC3 was set up and is presented, where a set of antennas operating at frequencies lower than $1 \mathrm{GHz}$ are combined into an array. The individual antennas cover the broadband frequency range from $10 \mathrm{MHz}$ to $1000 \mathrm{MHz}$.

12th European VLBI Network Symposium and Users Meeting

7-10 October 2014

Cagliari, Italy

\section{${ }^{1}$ Speaker}




\section{Introduction}

A project called AntArr with a new application of the DBBC3 [1] (Digital Base Band Converter, $3^{\text {rd }}$ generation) is presented. A group of antennas operating at low frequencies, in the range from $10 \mathrm{MHz}$ to $1000 \mathrm{MHz}$, are phased up for VLBI or pulsar observations. Moreover dedicated elements can be added to reach still lower frequencies to observe the range down to $\mathrm{kHz}$ frequencies. The DBBC3 manages the array operations in a selected portion of the band and the main characteristic is to synthesize a beam with an innovative approach. The final product of the array is a single station standard VLBI data stream for correlation with other antennas, or a synthesized beam for pulsar observations. Some antenna and array prototypes have been realized and are under test at two locations.

\section{Architecture}

The general architecture of the AntArr instrument is schematically indicated in the Fig.1b. Each green circular element represents a single antenna. More of such antennas are organized in arms as to form a matrix whose rows are subject to the progressive formation of the array signal that is finally forwarded to one of the inputs of the DBBC3. A maximum number of 32 inputs is available for a maximum number of 32 elements in a single arm, bringing the maximum number of elements of the array to 1024 .

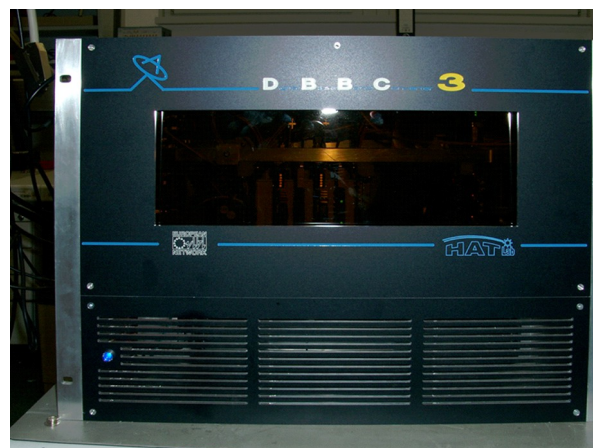

Fig 1a: DBBC front view.

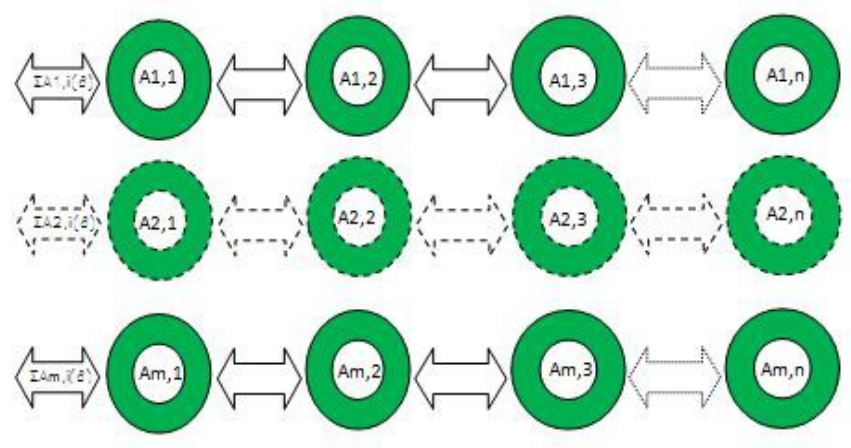

Fig 1b: AntArr schematic view.

\section{Array and Antennas}

An antenna array has been developed whose signals will be processed by the VLBI backend DBBC3L [2]. A set of antennas is organized in arms, in groups of four managed by a single ADB3L-CORE3L in order to create one or more synthesized beams. The signal from each antenna has the model delay applied to compensate the path length difference of the incoming radiation relative to its direct neighbour antenna. The delayed signal is summed to this next neighbour. The resulting signal has again the model delay applied relative to its next neighbour and the combined signal is added to it and so on, up to the DBBC3 processor. Any single beam is also available to be correlated with either the synthesized beam or with any other element in the array to precisely determine the delay residual with respect to the model of the single elements as a function of the time. The signal of the source is then tracked with a mix of 
theoretical geometric model and the residuals determined with the array itself. In Fig. 2 the prototype of an antenna is shown with its 3D beam in Fig. 3.

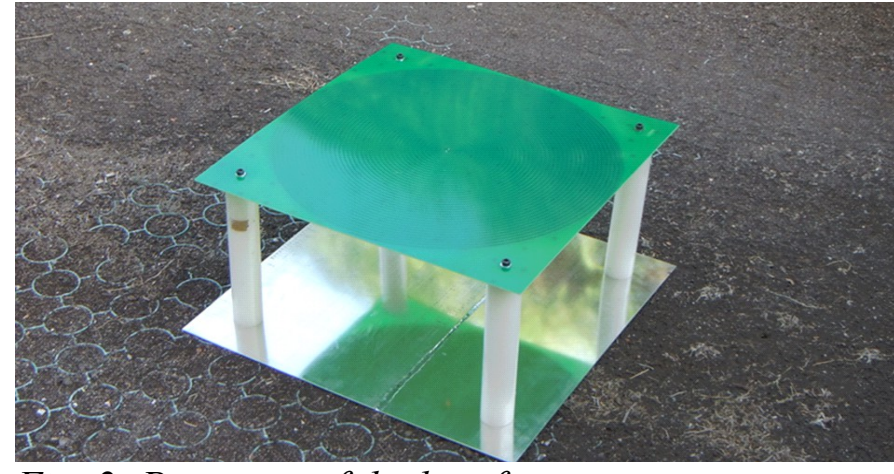

Fig. 2: Prototype of the low-frequency antenna

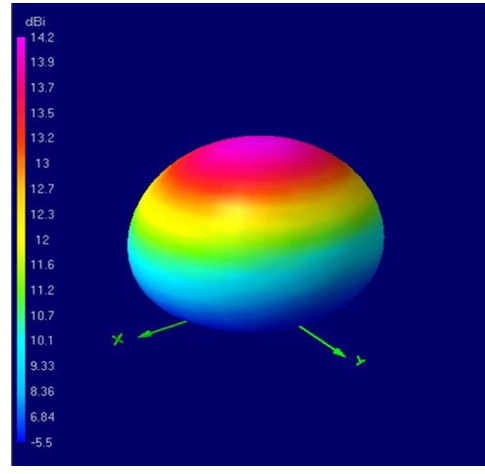

Fig. 3: Antenna beam

\section{General features of the Array and beam Synthesis}

Here you see an overview of the main features of the instrument:

- Antenna Prototype frequency range: $10 \mathrm{MHz}-1000 \mathrm{MHz}$

- Max. number of antennas in a single arm: 32

- Max. number of arms with a single DBBC3L: 32

- Max. observation bandwidth of each arm: $128 \mathrm{MHz}$

- Analogue delay compensation digitally controlled at every antenna

- Analogue summation for each arm at every antenna

- Digital correlation between any element of the array

- Digital correlation between any element of the array and the synthesized beam

\section{Stations and array characterization}

Two stations have been equipped for testing the network capability: one in the vicinity of the Noto radio telescope, the other on the northern slope of the Etna Volcano at low altitude $(600 \mathrm{~m})$. This last station will be operated with support of the Messina University. In the Fig. 4 and 5 a view of the relative position of the sites and one of the prototypes close to the Noto radio telescope are shown. 


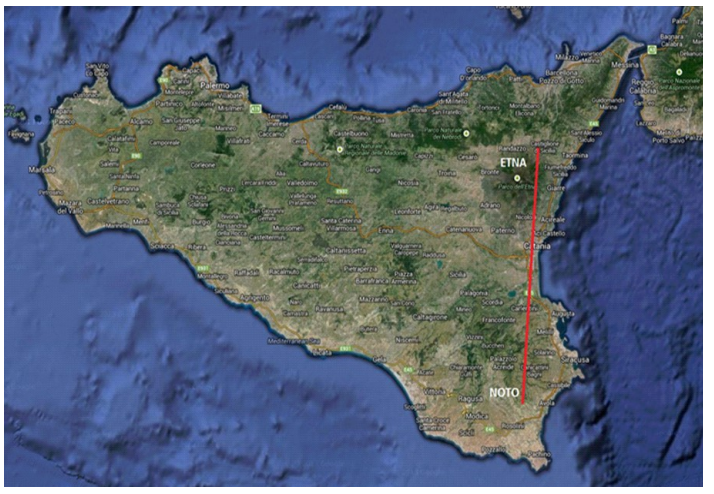

Fig. 5: Noto - Etna baseline, $114 \mathrm{~km}$.

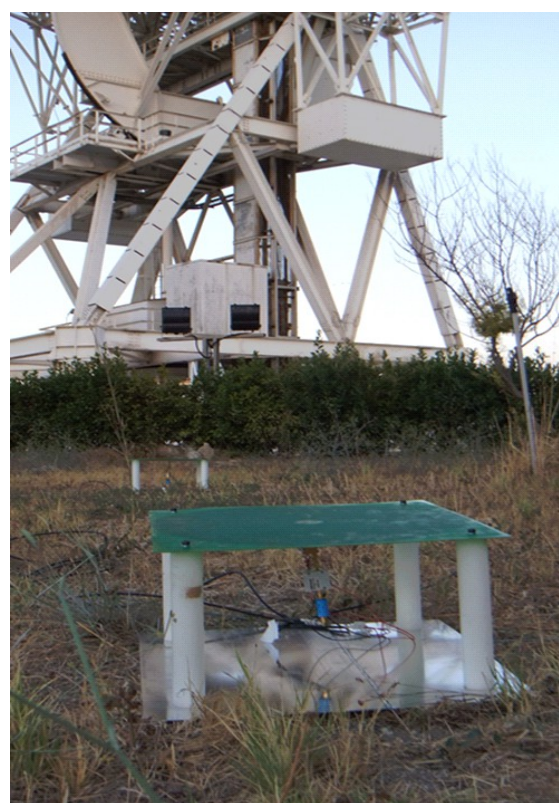

Fig. 6: Antenna prototype

A drone equipped with a multi-frequency transmitter is used to test and characterize the single antenna and groups of antennas in the far field regime. The vehicle can be placed in the sky at fixed positions controlled by an on-board GPS and an altimeter, in order to determine the 3D complex beam of a single antenna and the part of the array under analysis. This method allows to perform holographic measurements of the physical position of the single array element to make a map for the beam synthesis. The same drone with a different transmitter operating at the VLBI bands 22-43-86 GHz is under test for the holography phase reference measurements of the Noto radio telescope. This method looks promising for operating with VLBI receivers at different elevation angles. In Fig. 6 a picture of the drone is shown.

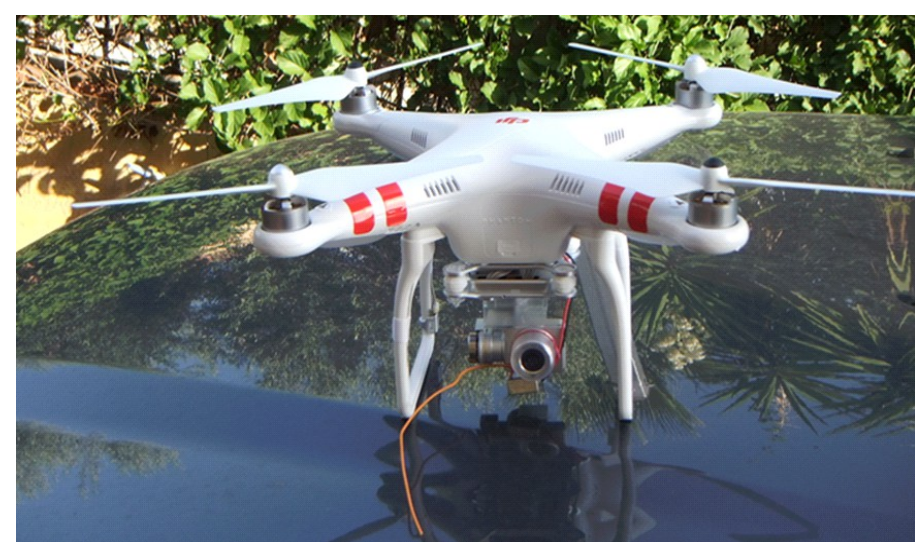

Fig. 6: Drone equipped with a transmitter for 327, 654, $981 \mathrm{MHz}$.

\section{References}

[1] G. Tuccari, DBBC3, Proceedings of the $20^{\text {th }}$ EVGA Meeting and $12^{\text {th }}$ Analysis Workshop, Bonn, Germany, pag. 19-21, 2011.

[2] G. Tuccari, W. Alef, A. Bertarini, S. Buttaccio, S. Casey, G. Comoretto, D. A. Graham,M. Lindqvist, A. Roy, J. Wagner, M. Wunderlich DBBC3: VLBI at 32 Gbits per second. 